\title{
Changes in Surfactant Phospholipids in Fetal Rat Lungs from Normal and Diabetic Pregnancies
}

\author{
MICHEL RIEUTORT, PHILIP M. FARRELL, MICHAEL J. ENGLE, \\ BERNADETTE PIGNOL, AND JACQUES R. BOURBON \\ Laboratoire de Physiologie du Developpement, College de France, Paris, France and Department of Pediatrics, \\ University of Wisconsin, Madison, Wisconsin 53792
}

\begin{abstract}
The purposes of this study were to adapt and evaluate further a pulmonary surfactant isolation method applicable to unperfused fetal rat lung, to quantitate key phospholipids phosphatidylcholine (disaturated phosphatidylcholine, and phosphatidylglycerol) of the isolated material during the last 3 days of gestation, and to determine if abnormalities in surfactant phospholipids were present in fetuses of diabetic pregnancies. A simplified scheme of sucrose gradient centrifugation proved useful for small scale preparations of material enriched in the phospholipids most characteristic of pulmonary surfactant. It was shown that fetal blood phospholipids did not contaminate the surfactant fraction and therefore would not produce artifacts in assessment of lung maturational changes. Analyses of subcellular fractions isolated at 19.5, 20.5 , and 21.5 days revealed that the percentages of disaturated phosphatidylcholine relative to total phospholipids were $23-44 \%$ in the surfactant preparations and 14$21 \%$ in the residual (nonsurfactant) fractions, while the disaturated phosphatidylcholine/phosphatidylcholine ra-
\end{abstract} tios were $0.62 \pm 0.06$ and $0.41 \pm 0.03$, respectively. Summation of the amounts of individual phospholipids in the two fractions yielded data that were nearly identical to the concentrations of these compounds in whole fetal lung samples analyzed independently, implying that losses during the surfactant isolation technique were negligible. The concentrations of phosphatidylcholine, disaturated phosphatidylcholine, phosphatidylglycerol, and total phospholipids increase markedly (more than 10-fold) and progressively in surfactant fractions prepared from normal fetal rat lung at $19.5,20.5$, and 21.5 days of gestation. In contrast, the residual fractions showed no changes from 19.5 to 20.5 days and then relatively modest increases from 20.5 to 21.5 days, except for phosphatidylglycerol, which increased markedly. The appearance of phosphatidylglycerol was first noted in the surfactant fraction at 20.5 days, but the level of this phospholipid did not show a marked increase until 21.5 days. These data are in agreement with previous morphologic and physiologic observations on fetal rat lung during late gestation and are also in keeping with clinical results from amniotic fluid analyses. Assessment of diabetic pregnancies revealed that at 20.5 days all phospholipids measured were significantly decreased in

Received September 19, 1985; accepted March 4, 1986

Address all correspondence and reprint requests to Dr. Philip M. Farrell, University of Wisconsin, Department of Pediatrics, Clinical Sciences Center, H4/ 458, 600 Highland Avenue, Madison, WI 53792.

This work was supported by NIH Pulmonary SCOR Grant P50-HL-27358 and I.N.S.E.R.M. Research Grant PRC 135002, Reproduction et Developpement. M.R. performed part of this study as a Hewlett Fellow of the University of Wisconsin P.M.F. as a NIH Fogarty Senior International Fellow (1-F06-TW00881-01), and M.J.E. as the recipient of NIH Research Career Development Award KD4-HD00350 the surfactant fraction, but not in the residual material. Neither surfactant nor residual phospholipids were decreased in diabetic pregnancies at 19.5 or 21.5 days of gestation. The transient nature of abnormal fetal lung surfactant phospholipids in diábetic pregnancies suggests impaired timing of the pulmonary maturation processes. (Pediatr Res 20: 650-654, 1986)

\section{Abbreviations}

RDS, respiratory distress syndrome PC, phosphatidylcholine

DSPC, disaturated phosphatidylcholine

PG, phosphatidylglycerol

ip, intraperitoneal

GTT, glucose tolerance test

$\mathrm{S}$, surfactant

$\mathbf{R}$, residual

Neonatal RDS occurs with an increased incidence in infants born prematurely to diabetic mothers whose hyperglycemia is not controlled during late gestation (1). Because RDS is associated with a deficiency of pulmonary surfactant phospholipids (2), it has long been suspected that fetal lung phospholipids characteristic of surfactant, such as PC and PG, are altered in diabetic pregnancies. Indirect evidence supporting this hypothesis has been obtained by analysis of human amniotic fluid and by some investigations of animal models of diabetes, as has been extensively reviewed by Bourbon and Farrell (3). Although altered lung phospholipid concentrations have been observed by many investigators, there are a number of discrepancies in results and uncertainties about whether PC and PG are abnormal in surfactant per se (3). Possible explanations for the discrepancies include variations in the animal species studied, the timing of gestation, the severity of maternal diabetes, and the biochemical approach used to quantitate fetal lung phospholipids. In the rat and rabbit, nevertheless, many observations converge to the conclusion that maternal diabetes delays morphologic (4-6) and biochemical (6-8) maturation of the fetal lung, making these species useful animal models when they are given pancreatic $\beta$ cell toxic agents, e.g. alloxan and STZ.

Except for recent studies of lung lavage fluid in fetuses near term gestation (6), most of the previous evaluations of fetal pulmonary phospholipids in diabetic rat and rabbit pregnancies have involved lipid extraction of whole lung tissue (3). Because of the lung's cellular heterogeneity and the low proportion of surfactant phospholipids relative to whole lung, much of the previous research on this problem has been somewhat lacking in sensitivity and specificity. Yet, methods for obtaining both intra- 
cellular and extracellular (i.e. alveolar) surfactant from salineperfused adult lung tissue were developed 16 yr ago by Frosolono et al. (9) and have proven more sensitive than whole lung analyses $(10,11)$. As pointed out by Frosolono (12), these techniques, which depend on density gradient centrifugation, are difficult to apply to small tissue samples. An additional problem encountered with perinatal lung is the difficulty of removing blood by perfusion because of the small fetal size and the high pulmonary vascular resistances and variable flows depending on the stage of development (13). Using Frosolono's method $(9,12)$ as modified by Sanders and Longmore (14), Engle et al. (15) succeeded in isolating from cultured fetal lung cells a subcellular fraction of phospholipids enriched in DSPC, the lipid most characteristic of pulmonary surfactant.

The objectives of this project were to adapt, simplify, and evaluate a modified surfactant isolation technique for application to fetal rat lung and to determine the phospholipid composition of pulmonary surfactant prepared from fetuses of normal and diabetic pregnancies that were carefully defined with respect to gestational age and glucose tolerance. Our goal was to determine to what extent the surfactant and nonsurfactant fractions were altered by the changes previously reported to occur during late gestation in total PL, PC, DSPC, and PG.

\section{MATERIALS AND METHODS}

Animals. Wistar rats (Centre d'Elevage R, Janvier, France) weighing approximately $200 \mathrm{~g}$ were used. They were given food and tap water ad libitum and maintained on a 14/10 h light/ dark cycle at $24^{\circ} \mathrm{C}$. Female rats were caged overnight with a male and mating was confirmed by examination of vaginal smears immediately after separation from the male. Ovulation, which occurs at $1 \mathrm{AM}$ on the average, was considered as the beginning of gestation; the following morning was denoted as 0.5 day of gestation. Term gestation is about 22 days under these conditions and varies throughout the year by no more than $12 \mathrm{~h}$.

Diabetes was induced in mated rats on day 0.5 of gestation by a single injection of $4 \%$ STZ (mixed D-L anomers, Sigma Co., St. Louis, MO) in 0.4 mol $\cdot$ liter $^{-1}$ citrate buffer ( $\mathrm{pH} \mathrm{4.2);} 28$ to $45 \mathrm{mg} / \mathrm{kg}$ body weight were administered in an attempt to obtain diabetes of graded severity. Sham-injected animals received an equivalent amount of buffer solution alone and their fetuses were used as controls. Characterization of the severity of diabetes was determined as previously described (6), including a standardized ip GTT. The results from these tests allowed the rats to be assigned to one of three diabetic subgroups: 1) subdiabetic, with normal or slightly increased post absorptive blood glucose (4.5 to 5.5. $\mathrm{mmol} \cdot$ liter $^{-1}$ ), without glucosuria, but with abnormal ip GTT; 2) mildly diabetic, with postabsorptive glucose ranging from 6 to $11.5 \mathrm{mmol} \cdot$ liter $^{-1}$, abnormal ip GTT and glucosuria; and 3) severely diabetic, with postabsorptive blood glucose over $16.5 \mathrm{mmol} \cdot$ liter $^{-1}$, high glucosuria and markedly abnormal ip GTT (6).

Fetal blood samples were taken to document glycemic condition and to verify that fetuses were representative of the three groups described in detail previously (6). Blood was taken from the axillary vessels under maternal sodium pentobarbital anesthesia between 9 and 10 AM either on day 19.5 , or 20.5 , or 21.5 of gestation, as previously described (6). After maximum bleeding of each fetus, fetal lungs were removed, frozen in liquid nitrogen, and stored at $-25^{\circ} \mathrm{C}$ until biochemical determinations were performed.

Surfactant isolation. Individual analyses of lung phospholipid composition were performed on fetuses at 21.5 days, but lungs from two to three fetuses were combined at either 19.5 or 20.5 days in order to obtain a satisfactory yield of surfactant material. With the present shortened isolation method, the treatment of as little as $70 \mathrm{mg}$ of tissue was possible at term gestation, when lungs are mature. The $S$ and nonsurfactant $(R)$ fractions of phospholipids were prepared by the method of Frosolono et al. (9) as modified by Sanders and Longmore (14) and then simplified as described below after verifying that results were the same with small lung tissue samples with or without washing the crude ("I band") as well as the purified ("IB band") $\mathrm{S}$ fractions. In preliminary experiments, the addition of blood was tested to evaluate the possible contamination of the $S$ fraction with blood phospholipids. Specifically, $120 \mu \mathrm{l}$ of fetal rat blood were added to one of two lung homogenates processed in parallel, each representing approximately $800 \mathrm{mg}$ of lung.

The lungs were weighed and gently homogenized in 0.154 mol.liter ${ }^{-1} \mathrm{NaCl}, 10 \mathrm{mmol} \cdot$ liter $^{-1}$ Tris $\mathrm{HCl}$ buffer ( $\mathrm{pH} 7.4$ ) with $1 \mathrm{mmol} \cdot \operatorname{liter}^{-1}$ EDTA. These and all procedures were performed at $4^{\circ} \mathrm{C}$. After a 10 -min centrifugation at $500 \times g$, the pellet was resuspended and homogenized twice. The two homogenates were pooled and then layered on a solution of $0.75 \mathrm{~mol} \cdot \mathrm{liter}^{-1}$ sucrose in the same buffer. After a $60 \mathrm{~min}$ centrifugation $\left(48,000 \times g_{\mathrm{av}}\right)$, the interfacial material (crude surfactant layer) between the supernatant and $0.75 \mathrm{~mol} \cdot$ liter $^{-1}$ sucrose was removed and layered over a discontinuous density gradient of 0.68 and $0.25 \mathrm{~mol}$. $\operatorname{liter}^{-1}$ sucrose. After a second centrifugation $(60 \mathrm{~min}, 63,000 \times$ $\left.g_{\text {av }}\right)$, the $\mathrm{S}$ fraction at the interface between the two sucrose concentrations was collected and stored at $-20^{\circ} \mathrm{C}$ until assayed. All material and sucrose solutions of the intermediary steps were gathered as the $\mathrm{R}$ fractions and stored at $-80^{\circ} \mathrm{C}$ until being homogenized for the phospholipid assays.

Biochemical analyses. Lipids were extracted overnight at $4^{\circ} \mathrm{C}$ from $\mathrm{S}$ or $\mathrm{R}$ fractions with chloroform-methanol using either the proportions recommended by Folch et al. (16) or the technique of Bligh and Dyer (17) which is especially useful for the larger volume $\mathrm{R}$ fraction. Approximately $20,000 \mathrm{dpm}$ of ${ }^{14} \mathrm{C}$ dipalmitoyl-phosphatidylcholine $\left(60 \mu \mathrm{Ci} \mu \mathrm{mol}^{-1}\right.$ purchased from Amersham, Paris, France) were added to each sample, allowing evaluation of losses of phospholipids during the assays. The phospholipids of the extracts were separated by two-dimensional thin-layer chromatography on silica gel $\mathrm{H}$ plates as described elsewhere (18). The various phospholipids were identified by two-dimensional reference to pure standards (Sigma). Each phospholipid spot, revealed by iodine vapor, was scraped from the plate and eluted from gel by the Bligh and Dyer (17) method. DSPC was isolated by the method of Mason et al. (19) on aliquots of the PC samples. After mineralization of the samples, inorganic phosphorus measured by the assay method of Ames and Dubin (20) served to determine the phospholipid concentration, expressed as nmol Pi per mg wet lung weight.

\section{RESULTS}

In our study of blood contamination, it was readily seen that the surfactant isolation procedure resulted in the hemoglobin being distributed in the residual fraction. The following phospholipid values (expressed as $\mathrm{nmol} / \mathrm{mg}$ ) were found in the surfactant fractions of the control and blood contaminated samples, respectively: $\mathrm{PC}=3.67$ and $3.61 ; \mathrm{DSPC}=1.72$ and $1.65 ; \mathrm{PG}=$ 0.274 and 0.226 . In normal fetal lung, the amount of blood phospholipid contaminating the residual fraction would be negligible.

Phospholipid data obtained for the surfactant and residual fractions are presented in Tables 1 and 2, respectively, and revealed a distinct difference in the composition of the two isolates. The surfactant fractions, irrespective of gestational age of group, always contained a higher proportion of DSPC than the comparable residual fractions. The percentages of DSPC relative to total phospholipids of the surfactant fractions ranged from 23 to $44 \%$, whereas the corresponding figures in the residual fractions were $14-21 \%$. The average DSPC/PC ratio of surfactant fractions was higher $(p<0.001)$ at $0.62 \pm 0.06$ (mean $\pm \mathrm{SD}$; range $=0.55-0.73$ ) than the ratio in parallel residual fractions $(0.41 \pm 0.03 ;$ range $=0.37-0.45)$. This degree of enrichment in DSPC was as expected from previous evaluations of the lipid 
Table 1. Surfactant phospholipids (nmol/mg wet lung tissue) in fetal lung from normal and diabetic rat pregnancies (mean \pm SD)

\begin{tabular}{|c|c|c|c|c|}
\hline Gestation group & $\begin{array}{c}\text { Total } \\
\text { phospholipids }\end{array}$ & $\mathrm{PC}$ & DSPC & PG \\
\hline \multicolumn{5}{|l|}{19.5 days } \\
\hline Normal $(6)^{*}$ & $0.746 \pm 0.130$ & $0.307 \pm 0.062$ & $0.170 \pm 0.032$ & 0 \\
\hline Diabetic (4) & $0.729 \pm 0.036$ & $0.241 \pm 0.115$ & $0.176 \pm 0.048$ & 0 \\
\hline \multicolumn{5}{|l|}{20.5 days } \\
\hline Normal (7) & $2.42 \pm 0.63 \dagger$ & $1.42 \pm 0.330 \dagger$ & $0.842 \pm 0.214 \dagger$ & $0.026 \pm 0.030$ \\
\hline Diabetic (9) & $\begin{array}{l}1.28 \pm 0.15 \\
(p<0.02) \ddagger\end{array}$ & $\begin{array}{c}0.918 \pm 0.301 \\
(p<0.01) \ddagger\end{array}$ & $\begin{array}{c}0.565 \pm 0.201 \\
\quad(p<0.02)\end{array}$ & $\begin{array}{c}0.004 \pm 0.005 \\
(p<0.05) \ddagger\end{array}$ \\
\hline \multicolumn{5}{|l|}{21.5 days } \\
\hline Normal (10) & $5.05 \pm 0.38 \dagger$ & $3.23 \pm 0.35 \dagger$ & $1.86 \pm 0.20 \dagger$ & $0.25 \pm 0.06 \dagger$ \\
\hline Diabetic $(32) \S$ & $\begin{array}{l}4.32 \pm 0.61 \\
(p<0.05) \ddagger\end{array}$ & $2.92 \pm 0.63$ & $1.81 \pm 0.27$ & $0.23 \pm 0.08$ \\
\hline
\end{tabular}

* No. of fetal lung samples analyzed.

† Statistically significant differences $(p<0.001)$ between groups of different gestational ages in the same experimental categories (control or diabetic rats)

$¥$ Statistical comparison between the normal and diabetic groups at the same gestational age.

$\S$ This group includes 11 subdiabetic, 11 mild diabetic, and 10 severe diabetic pregnancies.

Table 2. Nonsurfactant (residual fraction) phospholipids (nmol/mg wet lung tissue) in fetal lungs from normal and diabetic pregnancies (mean $\pm S D)^{*}$

\begin{tabular}{|c|c|c|c|c|}
\hline Gestation group & $\begin{array}{c}\text { Total } \\
\text { phospholipids }\end{array}$ & $\mathrm{PC}$ & DSPC & PG \\
\hline \multicolumn{5}{|l|}{19.5 days } \\
\hline Normal (6) & $12.9 \pm 0.68$ & $5.00 \pm 0.33$ & $1.86 \pm 0.23$ & $0.086 \pm 0.076$ \\
\hline Diabetic (4) & $12.7 \pm 1.07$ & $4.47 \pm 0.71$ & $1.96 \pm 0.45$ & $0.037 \pm 0.028$ \\
\hline \multicolumn{5}{|l|}{20.5 days } \\
\hline Normal (7) & $12.0 \pm 1.60$ & $4.95 \pm 1.15$ & $2.10 \pm 0.25$ & $0.091 \pm 0.068$ \\
\hline Diabetic (9) & $12.7 \pm 1.20$ & $5.51 \pm 0.94$ & $2.05 \pm 0.27$ & $0.065 \pm 0.059$ \\
\hline \multicolumn{5}{|l|}{21.5 days } \\
\hline Normal (6) & $14.7 \pm 2.59 \dagger$ & $7.25 \pm 2.03 \dagger$ & $3.00 \pm 0.32 \ddagger$ & $0.51 \pm 0.06 \ddagger$ \\
\hline Diabetic $(18) \S$ & $15.5 \pm 3.01$ & $7.30 \pm 1.77$ & $3.29 \pm 1.20$ & $0.43 \pm 0.08$ \\
\hline
\end{tabular}

* Statistical analyses were performed by unpaired Student's $t$ tests.

$\dagger p<0.05$, based on comparison of normal fetuses at 21.5 days with normals at 20.5 days.

$\ddagger p<0.001$, based on comparison of normal fetuses at 21.5 days with normals at 20.5 days.

$\S$ This group includes six fetuses from each of three diabetic subgroups (sub-, mild, and severe).

composition of surfactant material isolated from adult rats and dogs (21). In addition, near term when PG levels increased appreciably, the surfactant fraction phospholipids averaged $4.9 \%$ PG whereas the residual fraction had 3.5\%. A further quantitative assessment of the isolation method was made by observing that the summations of phospholipids in the surfactant and residual fractions yielded data comparable to values obtained independently by chloroform/methanol extraction of fetal rat whole lung samples. For instance, at term gestation, nearly identical DSPC and PC concentrations of 4.9 and $10.5 \mathrm{nmol} / \mathrm{mg}$ lung were obtained by the summation of $\mathrm{S}$ and $\mathrm{R}$ fractions, compared to 5.1 and $10.3 \mathrm{nmol} / \mathrm{mg}$ lung by direct analysis of whole lung (6).

The surfactant fractions (Table 1) showed progressive and highly significant (all $p<0.001$ ) increases in total phospholipids, PC, DSPC, and PG from 19.5 to 20.5 days of gestation and from 20.5 to 21.5 days. The most striking quantitative differences were in PC and DSPC which increased more than 10-fold in the $48 \mathrm{~h}$ after the 19.5-day baseline period, when we could first isolate a recognizable surfactant band between the sucrose layers. In contrast, the residual fractions showed no differences at 20.5 days compared to 19.5 days and, except for $\mathrm{PG}$, relatively modest increases in phospholipid concentrations during the final day of gestation. Alterations in PC and DSPC levels in whole fetal lung, therefore, are attributable solely to the surfactant fraction increases between 19.5 and 20.5 days and also largely to changes in this fraction at term gestation. At 20.5 days, the appearance of $P G$ was noted in the surfactant fractions from six of the seven normal lung samples (compared to four of nine diabetic preg- nancies). The concentration of $\mathrm{PG}$, however, showed the most impressive increase between 20.5 and 21.5 days. The net effect of these changes causes the surfactant fraction to contribute an increasing proportion to the whole lung phospholipid composition. Thus, surfactant fraction phospholipids, expressed as a percentage of the total phospholipids of whole lung (i.e. the summation of S and R fractions), account for $5.5 \%$ on day 19.5 , $16.8 \%$ on day 20.5 , and $26 \%$ at term; the last value is the same percentage found in adult rats (11).

As shown in Tables 1 and 2, assessment of fetal lung phospholipids in diabetic pregnancies compared to gestation-matched normal rats revealed no differences whatsoever at 19.5 days, no alterations in the residual fractions at any of the three gestational ages, and no abnormalities that could be ascribed to a separate diabetic subgroup, i.e. to a single category of diabetes severity. Between days 19.5 and 20.5, the lungs taken from fetuses of diabetic pregnancies showed much less increase than the normal fetuses in the level of surfactant fraction lipids. The diabetic group at 20.5 days showed significantly decreased total phospholipids $(p<0.02), \mathrm{PC}(p<0.01)$, DSPC $(p<0.02)$, and PG $(p$ $<0.05$ ) in the surfactant fraction as compared to controls (Table 1). The DSPC/PC ratio, however, was not decreased, nor was there a decrease in DSPC as a percentage of total phospholipids in the surfactant fraction. Thus, except for the absence of PG mentioned previously, the abnormality in surfactant phospholipids was predominantly of a quantitative rather than qualitative nature. Instead of the surfactant fraction phospholipids accounting for $16.8 \%$ of the total phospholipids of normal whole lung 
$(\mathrm{S}+\mathrm{R})$, fetuses of diabetic pregnancies showed an average of only $8.9 \%$ of total phospholipids in isolated surfactant on day 20.5. The PC and DSPC concentrations, however, rose substantially between 20.5 and 21.5 days in fetal lungs from all subgroups of diabetic pregnancies. Thus, there was very little difference near term between the normal and diabetic rats. At 21.5 days only the surfactant phospholipid concentration of the entire diabetic group remained lower than normal $(p<0.05)$ and accounted for a correspondingly lower percentage $(21.3 \%)$ of whole lung phospholipids. The concentration of PG in the surfactant and residual fractions were also similar to those of the normal fetuses studied at 21.5 days.

\section{DISCUSSION}

The method used herein for analysis of the major phospholipids of surfactant fractions prepared from fetal rats at 19.5 to 21.5 gestation gave results more specific than those of whole lung analyses and allowed access to some information on days 19.5 and 20.5 that would not be discernible with lung lavage. The values obtained for the various phospholipids measured in $S+$ $\mathrm{R}$ fractions (representing a total lung extract) are in good agreement with our previous data (6). This indicates that losses of lipid compounds are negligible with the method used herein. The higher DSPC/PC ratio found in $\mathrm{S}$ fractions compared to $\mathrm{R}$ fractions, as well as the surface active properties of $S$ fraction material (9), confirm the selectivity of the isolation method to prepare surfactant-enriched material.

It was of interest to find that striking increases occur in surfactant fraction phospholipids after 19.5 days of gestation, since osmiophilic lamellar bodies are first detectable then (22) and physiologic evidence of surfactant is not evident in short gestational species until the last 2 days of intrauterine development (23) (Pignol B, Bourbon J, unpublished data). This close association provides further support for the conclusion that the sucrose density gradient isolation procedure, first reported by Frosolono et al. (9) and then modified by others $(14,15)$, yields a subcellular fraction enriched in functionally significant phospholipids. The precise quantitation available with the surfactant isolation technique offers major advantages over the morphologic and physiologic assessments of fetal rat lung maturation. In comparison to whole lung lipid analyses, the approach reported herein offers greater sensitivity, as well as selectivity. In essence, the "background," nonsurfactant phospholipids, such as those in the plasma membranes and most subcellular organelles (12), are removed, allowing one to detect more readily the increases in surfactant phospholipids that signal fetal lung maturation.
The data of Tables 1 and 2, compared to the summation of $S$ $+\mathrm{R}$ phospholipids presented in Table 3 , indicate clearly that the marked changes in the surfactant fraction account for the somewhat subtle increases found in fetal rat whole lung analyses as reported previously (24). In fact, the changes in lung PC and DSPC levels from 19.5 to 20.5 days are entirely attributable to increases in the surfactant fraction. After 20.5 days, the further increase is also largely due to the surfactant fraction of fetal lung, but some of the newly synthesized DSPC and PG may be recovered in the endoplasmic reticulum and Golgi membranes that are presumably in the $\mathrm{R}$ fraction (12).

The small scale simplified technique of pulmonary surfactant isolation reported herein for unperfused fetal rat lung can be easily applied to developmental pulmonology research involving animal models, cultured tissue explants, and isolated pneumocytes, as long as the equivalent of $50-100 \mathrm{mg}$ of mature (i.e. term gestation) lung parenchyma are available. In this study we found that surfactant fraction assessment was useful in evaluating fetal lung development in streptozotocin-induced diabetic rat pregnancies. Under the conditions reported herein, fetuses from diabetic rats presented a transient delay in maturation of the surfactant material, particularly on day 20.5 , when all the phospholipids were significantly reduced. The decrease in total phospholipids was still observed in the $S$ fraction on the next day, while the PC and DSPC profiles were close to control values. The decrease in PG concentration observed in this study is consistent with previous data obtained with whole fetal lungs from the same diabetic animal model $(6,25)$ but the phenomenon was less pronounced. Surprisingly, the DSPC decrease observed in lung lavage specimens from term fetuses of sub- and mild diabetics (6) was not confirmed in the present work. Although no satisfying explanation can be given for this, there were differences in the biochemical methods used and minor variations in the precise timing of gestation. Furthermore, differences observed in lung lavage lipids (extracellular surfactant) when compared to the $\mathrm{S}$ fraction (extracellular plus intracellular surfactant) could be the consequence of processing of the phospholipids before the secretion of surfactant $(26,27)$ or to some impairment in secretion processes that might occur in fetuses of diabetic rats (6). These data also emphasize the importance of a precise timing of gestation in such studies. Several hours sooner or later might be sufficient to mask the changes in the phospholipid indices of lung maturation.

The advantages of surfactant fraction phospholipid quantitation become obvious when one observes that the residual fraction was not altered at any time in the diabetic group. Furthermore, summation of $S+R$ phospholipids, representing the equivalent of whole lung extraction, demonstrated that the significant de-

Table 3. Lung phospholipids (nmol/mg wet lung tissue) in fetal lungs from normal and diabetic pregnancies $(S+R$ fractions) $(\text { mean } \pm S D)^{*}$

\begin{tabular}{ccccc}
\hline & \multicolumn{3}{c}{ Total } \\
phospholipids & PC & DSPC & PG \\
Gestation group & & & & \\
\hline 19.5 days & $13.6 \pm 0.68$ & $5.30 \pm 0.36$ & $2.03 \pm 0.23$ & $0.087 \pm 0.076$ \\
$\quad$ Normal (6) & $13.2 \pm 0.92$ & $4.71 \pm 0.81$ & $2.13 \pm 0.41$ & $0.037 \pm 0.028$ \\
$\quad$ Diabetic (4) & $14.4 \pm 2.18$ & $6.38 \pm 1.36$ & $2.94 \pm 0.37 \dagger$ & $0.12 \pm 0.09$ \\
20.5 days & $14.4 \pm 1.55$ & $6.34 \pm 1.29$ & $2.61 \pm 0.34$ & $0.07 \pm 0.06$ \\
$\quad$ Normal (7) & $19.7 \pm 2.35 \ddagger$ & $10.5 \pm 2.05 \ddagger$ & $4.91 \pm 0.30 \S$ & $0.76 \pm 0.09 \S$ \\
Diabetic (9) & $20.3 \pm 3.07$ & $10.4 \pm 1.96$ & $5.09 \pm 1.18$ & $0.66 \pm 0.11$ \\
21.5 days & & &
\end{tabular}

\footnotetext{
* Statistical analyses were performed by unpaired Student's $t$ tests.

$\dagger p<0.001$, based on comparison of normal fetuses at 20.5 days with normals at 19.5 days

$\ddagger p<0.005$, based on comparison of normal fetuses at 21.5 days with normals at 20.5 days.

$\S p<0.001$, based on comparison of normal fetuses at 21.5 days with normals at 20.5 days.

$\|$ This group includes six fetuses from each of three diabetic subgroups (sub-, mild, and severe).
} 
creases in PC and DSPC at 20.5 days in the diabetic group would not have been detected without isolation of the surfactant fraction. This observation, as well as the transient nature of the abnormalities, helps to explain some of the discrepancies reviewed in detail elsewhere (3). It should be noted, however, that the pattern of abnormalities that we found in PC and DSPC is exactly the same as that reported by Gewolb et al. (8). Their data were obtained in a study of whole fetal lungs taken from streptozotocin rat pregnancies at 19-22 days of gestation, in which the low phospholipid levels were demonstrable only on day 21 which corresponds to day 20.5 as designated herein.

The fact that phospholipids in the fetal lung surfactant fraction increase at term gestation in the diabetic pregnancy agrees with the clinical hypothesis that the combination of maternal diabetes and premature delivery is what leads to the increased incidence of neonatal RDS (2). An alteration in the timing of fetal lung development caused by maternal diabetes, rather than in the fundamental nature of the maturation process, is in keeping with current concepts regarding the influence of hormones such as corticosteroids on fetal lung metabolism (28). An identical transient pattern of delayed pulmonary PC production just prior to term was observed previously with the anencephalic rat model using fetal lung slices (29). The mechanisms causing the abnormal timing of fetal lung development associated with maternal diabetes must still be delineated and include a great variety of possibilities (3), such as altered substrate utilization involving glycogen, free glucose, or fatty acids from triglycerides; impaired modulation by cyclic nucleotides; and direct inhibition of phospholipid synthesis by excessive levels of metabolites (e.g. myoinositol) or hormones (e.g. insulin).

Acknowledgments. The authors are grateful to Professor A. Jost for providing the research facilities at the College de France and to D. Jeannette Brown for expert technical assistance.

\section{REFERENCES}

1. Robert MF, Neff RK, Hubbell TP, Taeusch HW, Avery ME 1976 Association between maternal diabetes and the respiratory-distess syndrome in the newborn. N Engl J Med 294:357-360

2. Farrell PF, Avery ME 1975 Hyaline membrane disease. Am Rev Respir Dis 111:657-688

3. Bourbon JR, Farrell PM 1985 Fetal lung development in the diabetic pregnancy. Pediatr Res 19:253-267

4. Sosenko JRS, Frantz ID, Roberts RJ, Meyrick B 1980 Morphologic disturbance of lung maturation in fetuses of alloxan diabetic rabbits. Ann Rev Respir Dis 122:687-636

5. Tyden O, Berne C, Ericksson U 1980 Lung maturation in fetuses of diabetic rats. Pediatr Res 19:1192-1195

6. Bourbon JR, Pignol B, Martin L, Rieutort M, Tordet C 1985 Maturation of fetal rat lung in diabetic pregnancies of graduated severity. Diabetes 34:734743
7. Rhoades RA, Filler DA, Vannata B 1979 Influence of maternal diabetes on lipid metabolism in neonatal rat lung. Biochim Biophys Acta 572:132-138

8. Gewolb IH, Barrett C, Wilson CR, Warshaw JB 1982 Delay in pulmonary glycogen degradation in fetuses of streptozotocin diabetic rats. Pediatr Res 16:869-873

9. Frosolono MF, Charms BL, Pawlosvski R, Slivka S 1970 Isolation, characterization and surface chemistry of a surface-active fraction from dog lung. $J$ Lipid Res 11:439-457

10. Brown LAS, Bliss AS, Longmore WJ 1984 Effect of nutritional status on the lung surfactant system: food deprivation and caloric restriction. Exp Lung Res 6:133-147

11. Bruno JV, McMahon KE, Farrell PM 1985 Lung surfactant phospholipids as related to hydration and choline status of fasted rats. J Nutr 115:85-92

12. Frosolono MF 1982 Bronchoalveolar fluid and subcellular surfactant fractions. In: Farrell PM (ed) Lung Development: Biological and Clinical Perspectives, Vol 1. Academic Press, New York, pp 111-132

13. Olson EB Jr, Rankin J 1983 Isolated, perfused fetal rabbit lungs: preparation and flow relationships. Lung 161:87-98

14. Sanders RL, Longmore WJ 1975 Phosphatidylglycerol in rat lung. II. Comparison of occurrence, composition and metabolism in surfactant and residual lung fractions. Biochemistry 14:835-840

15. Engle MJ, Sanders RL, Douglas WHJ 1980 Type II alveolar cells in organotypic culture: model system for the study of surfactant synthesis. Biochim Biophys Acta 717:225-236

16. Folch J, Lees M, Sloane-Stanley GH 1957 A simple method for the isolation and purification of total lipides from animal tissues. J Biol Chem 226:497

17. Bligh EG, Dyer WJ 1959 A rapid method of total lipid extraction and purification. Can J Biochem Physiol 37:911-917

18. Bourbon JR, Rieutort M, Engle MJ, Farrell RM 1982 Utilization of glcogen for phospholipid synthesis in fetal rat lung. Biochim Biophys Acta 712:382382

19. Mason RJ, Nellenbogen J, Clements JS 1976 Isolation of disaturated phosphatidycholine with osmium tetroxide. J Lipid Res 17:281-289

20. Ames BN, Dubin DT 1960 The role of polyamines in the neutralization of bacteriophage deoxyribonucleic acid. J Biol Chem 235:769-775

21. Sanders RL 1982 The composition of pulmonary surfactant. In: Farrell PM (ed) Lung Development: Biological and Clinical Perspectives, Vol 1. Academic Press, New York, pp 193-210

22. Williams MCM, Mason RJ 1977 Development of the Type II cell in the fetal rat lung. Am Rev Respir Dis 115:37-47

23. Buckingham S, Avery ME 1962 Time of appearance of lung surfactant in the foetal mouse. Nature (Lond) 193:688-691

24. Farrell PM 1982 General features of phospholipid metabolism in the developing lung. In: Farrell PM (ed) Lung Development: Biological and Clinical Perspectives, Vol 1. Academic Press, New York, pp 223-238

25. Pignol B, Bourbon J, Rieutort M 1983 Diminution du phosphatidylglycerol pulmonaire chez les foetus de rattes renidues diabetiques par la streptozotocine. CR Acad Sci (Paris) Ser III 237:339-367

26. Jacobs H, Jobe A, Ikegami M, Conaway D 1983 The significance of reutilization of surfactant phosphatidylcholine. J Biol Chem 258:4156-4165

27. Magoon MW, Wright JR, Baritussio A, Williams MC, Goerke J, Benson BJ, Hamilton RL, Clements JA 1982 Subfractionation of lung surfactant: implications for metabolism and surface activity. Biochim Biophys Acta 750:18-31

28. Ballard PL 1982 Hormonal aspects of fetal lung development. In: Lung Development: Biological and Clinical Perspectives, Vol 2, Academic Press, New York, pp 205-253

29. Farrell PM, Blackburn WR, Adams A 1977 Lung phosphatidycholine synthesis and choline phosphotransferase activity in anencephalic rat fetuses with corticocosteroid deficiency. Pediatr Res 11:770-773 